\title{
Modeling of Optical Waveguide Poling and Thermally Stimulated Discharge (TSD) Charge and Current Densities for Guest/Host Electro Optic Polymers
}

\author{
Michael D. Watson, Paul R. Ashley, Mustafa Abushagur
}

\begin{abstract}
A charge density and current density model of a waveguide system has been developed to explore the effects of electric field electrode poling. An optical waveguide may be modeled during poling by considering the dielectric charge distribution, polarization charge distribution, and conduction charge generated by the poling field. These charge distributions are the source of poling current densities. The model shows that boundary charge current density and polarization current density are the major source of currents measured during poling and thermally stimulated discharge measurements. These charge distributions provide insight into the poling mechanisms and are directly related to $E_{\hat{A}}$ and , $\alpha_{\mathbf{r}}$ Initial comparisons with experimental data show excellent correlation to the model results.
\end{abstract}

Index Terms-Electrode Poling, Guest/Host Polymer, Mach-Zehnder Modulator, Waveguide, Thermally Stimulated Discharge Current ${ }^{1}$

\section{INTRODUCTION}

Poling of electro optic polymers is necessary to generate noncentrosymmetric chromophore structures possessing high electro optic coefficients. Much work has been done on

Manuscript received May 15, 2004. This work was supported through a Center Director's Discretionary Fund research effort at the NASA Marshall Space Flight Center and through the US Navy Air Warfare Center.

M. D. Watson is with the NASA Marshall Space Fight Center, Huntsville, AL 35812 USA (phone: 256-544-3186; fax: 256-544-094; e-mail: Michael.D.Watson (nasa.gov) and is currently pursuing a $\mathrm{PhD}$ in the Electrical and Computer Engineering Department of the University of Alabama in Huntsville.

P. R. Ashley is with the US Army AMRDEC, Huntsville, AL 35749 USA. (e-mail: P.R.Ashley@redstone.rdec.army.mil). M. Abushagur is Director of Microsystems-Engineering Research and Education at the Katie Gleason College of Engineering, Rochester Institute of Technology, Rochester, NY 14623 USA, (e-mail: mabushagur@rit.edu). studying the molecular behavior of dipoles and charge distributions in single layer polymer films as can be seen in several excellent books on the subject. $[1,2,3]$ This body of work has shed light on the molecular processes at work in polymer film poling and has considered both polar and non-polar films. These techniques have focused on a single layer with some limited work on 2 layer non polar films. Basic charge models have been developed for single layer and 2 layer nonpolar films $[1,4]$. The charge distribution and motion induced by ion beam poling and corona poling across air gaps have been studied extensively. [5,6] Studies based on dielectric constant measurements have also been conducted. [7] These polymer films have been studied by a variety of methods including Thermally Stimulated Discharge (TSD)[4], Second Harmonic Generation (SHG), pyroelectric currents, [8] and thermal and acoustic waves. $[9,10]$ Work has been reported on single layer guest/host systems [11] and for single layer polyamide film irradiation influence studies [12] using TSD for experimental analysis. Some limited work has been done on 3 layer planar film TSD currents. [13] Studies of waveguide structures have recently addressed the conductivity of the cladding layers [14], but these studies have not considered the boundary and polarization charge layers formed in a waveguide structure.

Optical waveguides are 3 layer structures consisting of an electro optic polymer bound by non polar polymer layers. These structures are laterally confined as well, complicating the poling region geometry. These devices are practically poled by contact electrodes, generally consisting of sputtered gold films on the outer cladding layers. A model is needed of the poling process in a 3-dimensional, 3 layer film consisting of both polar and non polar films. 
The molecular theory development, single layer charge models, and measurement techniques have provided a tremendous amount of knowledge on polymer films but are often difficult to employ in standard fabrication processes. Poling currents offer a straight forward macroscopic quantity that is easily measured during device fabrication. Poling and TSD current techniques offer a method to study material poling mechanisms including the activation energy, $E_{A}$, and the natural frequency, $\alpha_{\mathrm{r}}$, of the material dipoles. These currents are based on the flow of charge in the waveguide structure and thus a model has been developed to investigate the charge distribution and induced currents during the poling process. This paper develops the polarization and boundary charge model for an optical waveguide structure incorporating an Electro Optic guest/host polymer core.

Following this introduction, the waveguide poling model is developed in Section II. Section III provides a discussion of the model simulations and their comparison with some experimental data. Section IV provides the summary and conclusions.

\section{Waveguide Poling Model}

\section{A. Description}

Poling of a material stack can be described in terms of the charge densities and current densities produced through the poling process. Charge distributions within the dielectric layers lead to a boundary charge density along the boundaries between layers with differing dielectric constants. These represent MaxwellWagner boundaries In addition, polarization charge densities exist within polar layers. The motion of these charge densities leads to the material current densities and are directed by the poling field which also provides a conduction current density within the layers. These charge densities and current densities are determined by the layer material properties. Boundary charge densities are determined by the differences in layer conductivities and dielectric constants. Polarization charge densities are related to the alignment of dipoles. These alignments are related to the dipole activation energy $\left(E_{A}\right)$ and relaxation frequency $\left(\alpha_{4}\right)$. Activation energy is the energy required to orient a molecule. The $E_{A}$ is strongly dependent on the molecular environment surrounding the dipole. Natural frequency is a function of molecular shape and size.[4] For guest/host polymer systems, the chromophores are all of similar shape and size, so the natural frequency should be a constant. However, the local molecular cnvironment can vary greatly, depending on where the chromophore molecule is located with relationship to host molecule segments, the compactness of the host molecules, and the chromophore loading density. Thus, for guest/host polymer systems, distributions are expected in terms of the chromophore activation energy.

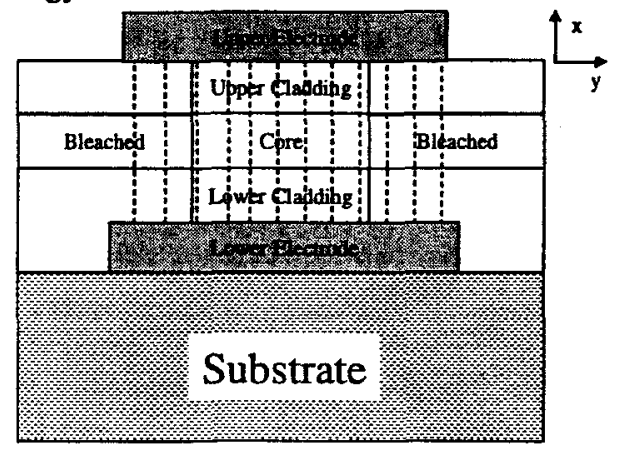

Fig. 1. Photo Bleached Waveguide Poling Geometry

Fig. 1 illustrates the typical photo bleached waveguide geometry. This geometry consists of 2 separate nonpolar polymer films sandwiching a polar guest/host polymer. Photo bleaching forms a narrow region in the core film providing lateral optical confinement. The photo bleaching destroys the chromophore molecules in the exposed region leaving only the waveguide core containing chromophore. Thus, photobleached waveguides can be modeled by noting that only the core region will have dipole orientations. The bleached core region can then be treated as a nonpolar material. Assuming diffusion is not a strong factor in TSD [4] the bleached regions will not contribute significantly to the discharge current. These regions have a similar molecular structure to the core, missing only the guest molecule. When poled, the dipoles in the bleached region are aligned parallel to the electric field as shown in Fig. 1. Both negative and positive charges exist along the bleaching boundary and the surface retains a neutral charge. Thus, the bleaching boundary does not form a Maxwell Wagner boundary.

Between the 3 film layers, Maxwell Wagner boundaries do exist. For optical waveguides the poling region and therefore the electrodes are typically confined to the waveguide region. Thus, no significant poling field exists outside the waveguide core and a rectangular geometry can be assumed for the model as shown in Fig. 1. 
Based on this assumption, no conduction charge is expected to be created in the bleached (nonpolar) core region. Thus, a 3 layer model can be employed with Maxwell Wagner charge boundaries between the upper cladding and core and between the core and lower cladding.

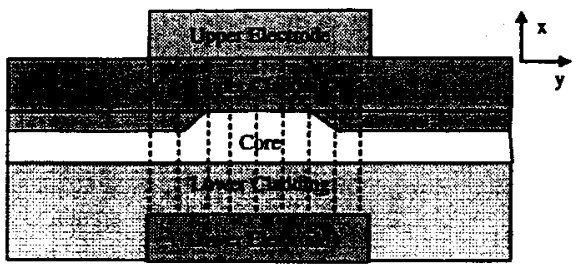

\section{Fig. 2. Rib Waveguide Poling Geometry}

Rib waveguides, as illustrated in Fig. 2, have a more challenging structure, but the same assumptions apply. During poling, only the waveguide core region is poled. Thus, there is a boundary between poled and unpoled core regions. Again, however, this boundary does not produce a charge surface, but merely represents a transition from vertically oriented dipoles to randomly oriented dipoles. Because the poling field is confined to the waveguide core region, and no conduction charge is generated outside the poling field. Thus rib waveguides, as well as trench waveguides which can be viewed as inverted ribs, can be represented by a 3 layer model with Maxwell Wagner charge boundaries between the upper cladding and core and between the core and lower cladding.

\section{B. Poling and Cool Down Equations}

The initial model will be developed following the original approach employed by J. Turnhout for a 2 layer nonpolar planar structure and a polar layer separated by an air gap from the upper electrode. [4] The most general waveguide case is the asymmetric waveguide: a polar layer sandwiched by 2 different nonpolar layers. Equations for the charge density and polarization can be derived beginning with the current density for a 3 layer stack. The single relaxation Debye Equation can then be employed to form a set of coupled differential equations.

A DC voltage source is connected across the waveguide electrodes for poling. No space charge is assumed to be stored prior to poling. The current density through each layer must be equal following Kirchoff's Laws. The poling field directs all current flows and dipole alignments in the same direction. So, the current density relationship can be written employing Gauss Law $\left(\mathrm{q}_{\mathrm{L}} / \mathrm{A}^{2}=\varepsilon_{0} \varepsilon_{\mathrm{L}} \mathrm{E}_{\mathrm{L}}\right)$ as:

$$
\begin{aligned}
& J(t)=\varepsilon_{o} \varepsilon_{c} \frac{d E_{c}(t)}{d t}+\gamma_{c}(T) E_{c}(t)= \\
& \varepsilon_{o} \varepsilon_{g} \frac{d E_{g}(t)}{d t}+\gamma_{g}(T) E_{g}(t)+\frac{d P_{s}(t)}{d t}, \\
& =\varepsilon_{o} \varepsilon_{d} \frac{d E_{s}(t)}{d t}+\gamma_{s}(T) E_{s}(t) \\
& \text { where, }
\end{aligned}
$$

$\varepsilon_{0}$ is the permittivity of free space, $\varepsilon_{c}, \varepsilon_{g}, \varepsilon_{s}$ are the dielectric constants for the upper cladding, waveguide core (guiding region), and lower cladding respectively,

$\gamma_{c}, \gamma_{g}, \gamma_{s}$ are the material conductivity of the upper cladding, waveguide core (guiding region), and lower cladding respectively,

$E_{c}(t), E_{g}(t), E_{s}(t)$ are the electric fields in the upper cladding, waveguide core (guiding region), and lower cladding respectively, dipoles.

$P_{s}(t)$ is the persistent polarization of the

Similarly, the voltage relationship is:

$$
\begin{aligned}
& V_{P}=E_{c}(t) h_{c}+E_{g}(t) h_{g}+E_{s}(t) h_{s}, \\
& \text { where } \\
& \mathrm{V}(\mathrm{t})=\mathrm{E}(\mathrm{t}) \mathrm{h} \\
& \mathrm{V}_{\mathrm{P}} \text { is the poling voltage } \\
& \text { and } \mathrm{h}_{\mathrm{c}}, \mathrm{h}_{\mathrm{g}}, \mathrm{h}_{\mathrm{s}} \text { are the thicknesses of the }
\end{aligned}
$$

upper cladding, waveguide core (guiding region), and lower cladding respectively.

Now Ohm's Law, $V(t)=i(t) / \gamma(T)$, can be applied assuming that the upper and lower claddings differ only in their resistivity so that the currents in these layers are equal. The surface charge along the Maxwell Wagner boundaries between the upper cladding and core can also be written using Gauss Law as:

$$
\sigma_{c g}(t)=\varepsilon_{0} \varepsilon_{g} E_{g}(t)+P_{s}(t)-\varepsilon_{0} \varepsilon_{c} E_{c}(t)
$$

From these assumptions, $\mathrm{Vg}(\mathrm{t})$ can then be written as:

$$
V_{g}(t)=\frac{\sigma_{c g}(t)-P_{s}(t)+\frac{\varepsilon_{0} \varepsilon_{c}}{h_{c}} V_{P}}{C_{s t}}
$$

where,

$$
\begin{gathered}
C_{s t}=\frac{\varepsilon_{0} \varepsilon_{g}}{h_{g}}+\frac{\varepsilon_{0} \varepsilon_{c}}{h_{c}} \Gamma_{c s}(T), \\
\Gamma_{c s}(\mathrm{~T})=\left(\frac{1}{1+\frac{\gamma_{c}(\mathrm{~T})}{\gamma_{s}(\mathrm{~T})}}\right) .
\end{gathered}
$$


The units of $C_{s t}$ are in $F / \mathrm{m}^{2}$ which represents a capacitance term for the stack while $\Gamma_{c s}$ is a dimensionless conductivity ratio.

Using the first 2 equalities from (1) and with $V_{g}(t)$ expanded by (4), equations can be written for $\mathrm{J}(\mathrm{t})$ and $\mathrm{d} \sigma_{\mathrm{cg}} / \mathrm{dt}$ as:

$J(t)=\left(\begin{array}{l}\frac{\gamma_{g}(T)}{h_{g} C_{s t}}\left(\sigma_{c g}(t)-P_{s}(t)+\frac{\varepsilon_{0} \varepsilon_{c}}{h_{c}} \Gamma_{c s}(T) V_{p}\right) \\ +\frac{\varepsilon_{0} \varepsilon_{g}}{h_{g}} \frac{d \sigma_{c g}(t)}{d t}+\frac{\varepsilon_{0} \varepsilon_{c}}{h_{c}} \Gamma_{c s}(T) \frac{d P_{s}(t)}{d t}\end{array}\right)$.

$\frac{d \sigma_{c g}(t)}{d t}+\Gamma_{g c}(T) \beta_{w}(T) \sigma_{c g}(t)=\Gamma_{g c}(T) \beta_{w}(T) P_{s}(t)$,

$+\beta_{w}(T) \Gamma_{c s}(T)\left(\frac{\varepsilon_{0} \varepsilon_{g}}{h_{g}}+\frac{\varepsilon_{0} \varepsilon_{c}}{h_{c}}\left(\Gamma_{c s}(T)-\Gamma_{g c}(T)\right)\right) V_{P}$

where,

$$
\begin{aligned}
& \Gamma_{\mathrm{gc}}(\mathrm{T})=\left(1+\frac{\mathrm{h}_{\mathrm{c}} \gamma_{\mathrm{g}}(\mathrm{T})}{\mathrm{h}_{\mathrm{g}} \gamma_{\mathrm{c}}(\mathrm{T})}\right) \\
& \beta_{w}(T)=\left(\frac{\gamma_{c}(T)}{h_{c} C_{s t}}\right)
\end{aligned}
$$

Now (8) gives an equation in 2 unknowns, $\sigma_{c g}(t)$ and $P_{s}(t)$. A second equation can be found for $P_{s}(t)$ by using (4) in the Debye Equation given by Turnhout. [4] The single relaxation frequency Debye equation is given by:

$$
\begin{aligned}
& \frac{d P_{s}(t)}{d t}+\alpha(T) P_{s}(t) \\
& =\varepsilon_{0}\left(\varepsilon_{\text {slow }}-\varepsilon_{g}\right) \alpha(T) E_{g}(t)
\end{aligned}
$$

Substituting $E_{g}(t)=V_{g}(t) / h_{g}$ and using (4) for $\mathrm{V}_{\mathrm{g}}(\mathrm{t})$ yields:

$$
\begin{aligned}
& \left(\frac{h_{c} \varepsilon_{g}}{\varepsilon_{c}}+h_{g} \Gamma_{c s}(T)\right) \frac{d P_{s}(t)}{d t} \\
& +\alpha(T)\left(h_{g} \Gamma_{c s}(T)+\frac{h_{c} \varepsilon_{s l o w}}{\varepsilon_{c}}\right) P_{s}(t) \\
& =\left(\varepsilon_{s l o w}-\varepsilon_{g}\right) \alpha(T)\left(\varepsilon_{0} V_{P}+\frac{h_{c}}{\varepsilon_{c}} \sigma_{c g}(t)\right)
\end{aligned}
$$

where $\varepsilon_{\text {slow }}$ is the low frequency dielectric response of the core material and $\alpha(T)$ is the dipole relaxation frequency.

Equations (8) and (12) form a coupled set of differential equations which can be solved to give the boundary charge density, $\sigma_{\mathrm{cg}}(\mathrm{t})$, and the polarization, $P_{s}(t)$. These equations apply to both the poling phase and the cool down phase while the voltage is still applied to the waveguide stack.

The ultimate value of these equations can bc derived assuming that the polarization and boundary charge densities cannot grow infinitely, but reach some maximum value during charging. This corresponds to saturation of charge sites able to be occupied by the boundary charge and orientation of all dipoles in the core layer. For these conditions, the charge densities are constant and the time derivative terms in (8) and (12) are zero. Thus, there are now 2 equations with 2 unknowns $\left(\sigma_{\mathrm{cg}}(\infty)\right.$ and $\operatorname{Ps}(\infty))$ which can be solved to yield:

$$
\begin{aligned}
& \sigma_{c g}(\infty)= \\
& {\left[\begin{array}{c}
\frac{\Gamma_{c s}(\infty)}{\Gamma_{g c}(\infty)}\left(\frac{\varepsilon_{0} \varepsilon_{g}}{h_{g}}+\frac{\varepsilon_{0} \varepsilon_{c}}{h_{c}}\left(\Gamma_{c s}(\infty)-\Gamma_{g c}(\infty)\right)\right) \\
\frac{h_{c} \varepsilon_{g}}{\varepsilon_{c}}+h_{g} \Gamma_{c s}(\infty) \\
\times\left(h_{g} \Gamma_{c s}(\infty)+\frac{h_{c} \varepsilon_{s l o w}}{\varepsilon_{c}}\right)+\frac{\varepsilon_{0}\left(\varepsilon_{s l o w}-\varepsilon_{g}\right)}{\frac{h_{c} \varepsilon_{g}}{\varepsilon_{c}}+h_{g} \Gamma_{c s}(\infty)}
\end{array}\right]}
\end{aligned}
$$

$$
\begin{aligned}
& P_{s}(\infty)=\left[\begin{array}{l}
\frac{1}{\frac{h_{c} \varepsilon_{g}}{\varepsilon_{c}}+h_{g} \Gamma_{c s}(\infty)}+\frac{\Gamma_{c s}(\infty)}{\Gamma_{g c}(\infty)} \\
\varepsilon_{0}\left(\varepsilon_{s l o w}-\varepsilon_{g}\right) \\
\left(\frac{\frac{\varepsilon_{g} h_{c}}{\varepsilon_{c} h_{g}}+\Gamma_{c s}(\infty)-\Gamma_{g c}(\infty)}{\frac{h_{c} \varepsilon_{g}}{\varepsilon_{c}}+h_{g} \Gamma_{c s}(\infty)}\right)
\end{array}\right] V_{P} .
\end{aligned}
$$

$\sigma_{c g}(\infty)-P_{s}(\infty)=$

$\left[\frac{\Gamma_{c s}(\infty)}{\Gamma_{g c}(\infty)}\left(\frac{\varepsilon_{0} \varepsilon_{g}}{h_{g}}+\frac{\varepsilon_{0} \varepsilon_{c}}{h_{c}}\left(\Gamma_{c s}(\infty)-\Gamma_{g c}(\infty)\right)\right)\right] V_{P}$

These equations show that both charge densities are linearly proportional to the poling voltage and also depend on the material properties (dielectric constants and conductivities) and waveguide geometry (thickness). Thus, dipole orientation efficiency is linearly proportional to the poling field. Equation (15) shows the difference between the maximum values is a constant as well. Note, that the polarization charge density can be seen as driven by the boundary charge density as will be clear from the experimental section below. Also, if the material conductivities for each layer vary similarly with temperature, then $\Gamma_{\mathrm{cs}}$ and $\Gamma_{\mathrm{gc}}$ will be constants across all temperatures. 
If a symmetric waveguide is assumed where the upper and lower cladding layers are the same matcrial and thickness, then the model also shows that the boundary layer at the upper cladding - core boundary and lower cladding core boundary are equal but of opposite charge: $\sigma_{\mathrm{cg}}(\mathrm{t})=-\sigma_{\mathrm{gs}}(\mathrm{t})$.

\section{TSD Equations}

During Thermally Stimulated Discharge (TSD), the waveguide electrodes are grounded so that $V p=0$. Solving (7), (8), and (12) for this condition provides the charge densities, and released current densities during the TSD phase. Following the same approach as for the charging phase yields the following equations for $P_{s}(t)$, $\sigma_{\mathrm{gc}}(\mathrm{t})$, and $\mathrm{J}_{\mathrm{r}}(\mathrm{t})$.

$$
\begin{aligned}
& \beta_{T S D}(T)=\frac{\gamma_{g}(T)}{h_{g} C_{s t}}+\frac{\gamma_{c}(T)}{h_{c} C_{s t}} \Gamma_{c s}(T) \\
& \frac{d \sigma_{c g}(t)}{d t}=\beta_{T J D}(T)\left(P_{s}(t)-\sigma_{c g}(t)\right) \\
& \frac{d P_{s}(t)}{d t}= \\
& \alpha(T)\left(\begin{array}{l}
\frac{\varepsilon_{0}}{h_{g} C_{s t}}\left(\varepsilon_{s l o w}-\varepsilon_{g}\right) \sigma_{c g}(t) \\
-\frac{1}{C_{s t}}\left(\frac{\varepsilon_{0}}{h_{g}}\left(\varepsilon_{s l o w}-\varepsilon_{g}\right)+\frac{\varepsilon_{0} \varepsilon_{g}}{h_{g}}+\frac{\varepsilon_{0} \varepsilon_{c}}{h_{c}} \Gamma_{c s}(T)\right) P_{s}(t)
\end{array}\right) \\
& J_{r}(t)=\frac{\varepsilon_{0} \varepsilon_{g}}{h_{g} C_{s t}} \frac{d \sigma_{c g}(t)}{d t}+\left(1-\frac{\varepsilon_{0} \varepsilon_{g}}{h_{g} C_{s t}}\right) \frac{d P_{s}(t)}{d t} \\
& +\frac{\gamma_{g}}{h_{g} C_{s t}}\left(\sigma_{c g}(t)-P_{s}(t)\right)
\end{aligned}
$$

\section{MODEL SIMULATION AND EXPERIMENTAL RESULTS}

Using these equations a model has been programed in Matlab to investigate various 3 layer systems. The systems modeled are a stack consisting of a Norland Optical Adhesive (NOA) 71 upper and lower claddings and a CLD1/APC (Amorphous Polycarbonate) core layer. Each layer was modeled as $3 \mu \mathrm{m}$ thick. Equations (7), (8) and (12) where used to describe a system with a single value of $\alpha_{1}$ and $E_{A}$. Fig. 3 shows the polarization and the boundary charge density build up in a 3 layer waveguide structure. Note that because of cross linking, the NOA71 $\mathrm{T}_{\mathrm{g}}$ cannot be determined specifically and ranges from $50 \mathrm{C}$ to $100 \mathrm{C}$ depending on processing steps. A value of $78 \mathrm{C}$ was assumed based on the modulator fabrication process. Similarly, the $\mathrm{T}_{\mathrm{g}}$ for CLD1/APC is dependent on the \%wt of the chromophore in the material and the quality of the dispersement of the CLD1 in the APC and cannot be determined specifically. A value of $141 \mathrm{C}$ was assumed based on the $\mathrm{T}_{\mathrm{g}}$ of APC, the $T_{g}$ of CLD1, and \%wt of the chromophore used in fabrication.

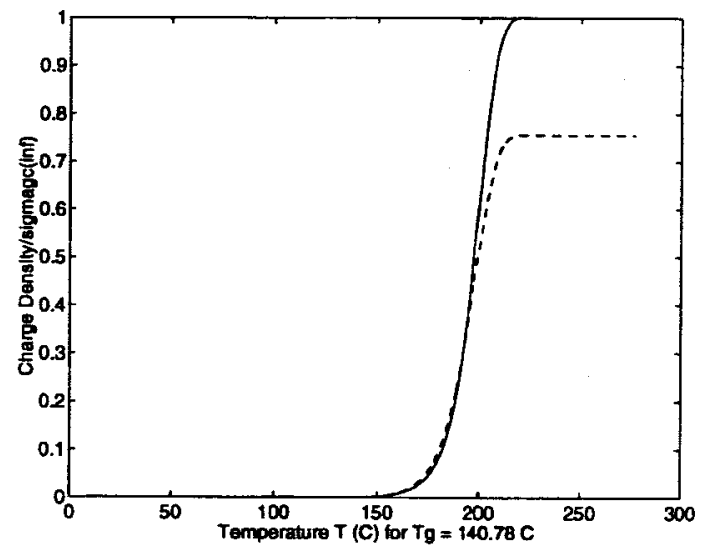

Fig. 3. Boundary Charge Density (+ line) and Polarization Charge Density (dashed line) during Poling

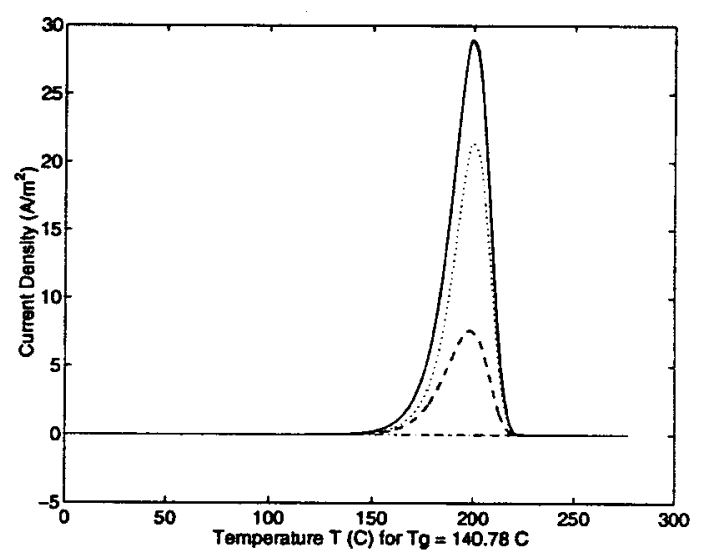

Fig. 4. Poling Current Densities. Total-solid Line, Boudnary - dotted line, dashed - polarization, Conduction - dot/dash line.

To model the relaxation frequency, an arrenhius function was used for a single activation energy and single relaxation frequency approximation. Various functions have been proposed to describe the relaxation frequency as a function of temperature. These include the WilliamsLandel-Ferry (WLF), and the WLF with an effective temperature to adjust the curve below Tg. $[1,15]$. However, for studies of poling phenomena at poling temperatures, the simple arrenhius function has proven fairly accurate. 
$[1,7,16]$ For the current modeling efforts, the arrenhius function has proven very accurate in generating poling charge and current relationships for the waveguide structure as can be seen from the experimental comparison below.

Equation (7) can be decomposed into 3 components: Polarization current, boundary charge current, conduction current. Fig. 4 shows how these 3 components and the total current charge density changes with temperature. For comparison with experimental data, very high heating ( $44 \mathrm{C} / \mathrm{min})$ and cooling $(14 \mathrm{C} / \mathrm{min})$ rates were used.

Both the boundary and the polarization charge densities produce current densities as they grow to their maximum values. Another interesting feature is the conduction current density, which is much smaller than the boundary charge and polarization charge current densities in the 3 layer model. For a guest/host system, the conduction current density is related to the conductivity of the host material since the guest chromophore is widely dispersed in a good quality film and is not attached to the host. Thus, the conduction current increases as the material temperature approaches the $T_{8}$ of the host APC and is not significant at the lower values of the combined guest/host $T_{B}$.

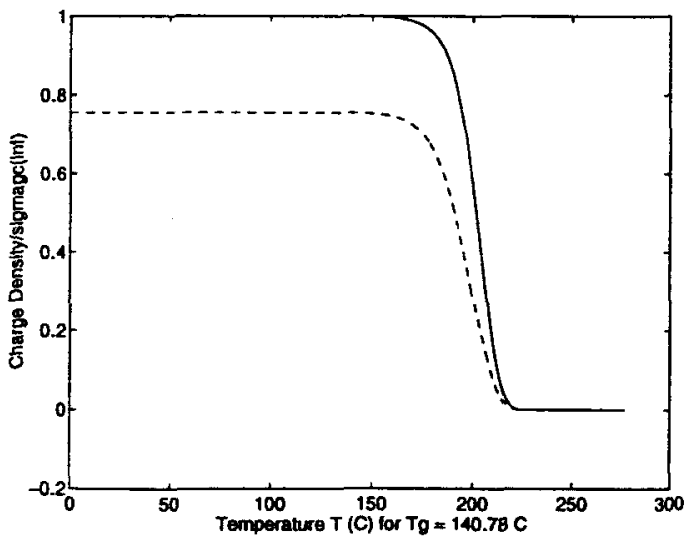

Fig. 5. Boundary Charge Density (solid line) and Polarization Charge Density (dashed line)densities during TSD.

During cool down, (7), (8), and (12) were again solved in Matlab, but with the final boundary charge density and polarization charge density reached during poling as initial conditions. Both charge densities remained constant at the level achieved in heating. Both the polarization and boundary charge current remain at zero indicating no change in the charge density and the charge is maintained during cool down.

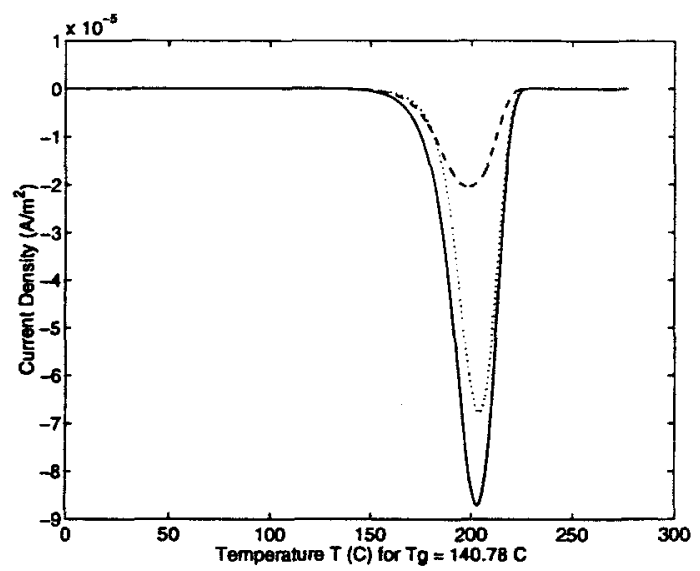

Fig. 6. TSD Current Densities. Total - solid Line, Boudnary - dotted line, dashed - polarization.

Fig. 5 illustrates the TSD case. Equations (17) (19) were solved in Matlab using the values of the boundary charge density and polarization charge density at the end of cool down as initial conditions. In this case the upper and lower electrodes are grounded. As the modulator is heated, the boundary charge density and polarization charge density decay to zero. Fig. 6 shows that the current density follows the decay of the charge densities with peaks corresponding to the release of each charge density. Note also that the magnitude of the released charge density is less than the poling charge density. Essentially, the poling field provides additional carriers which multiply the current densities. During TSD, only the charge induced fields are present which are much smaller than the poling field. This is readily seen be dividing the poling charge density magnitude by the poling field which yields the TSD current density magnitude.

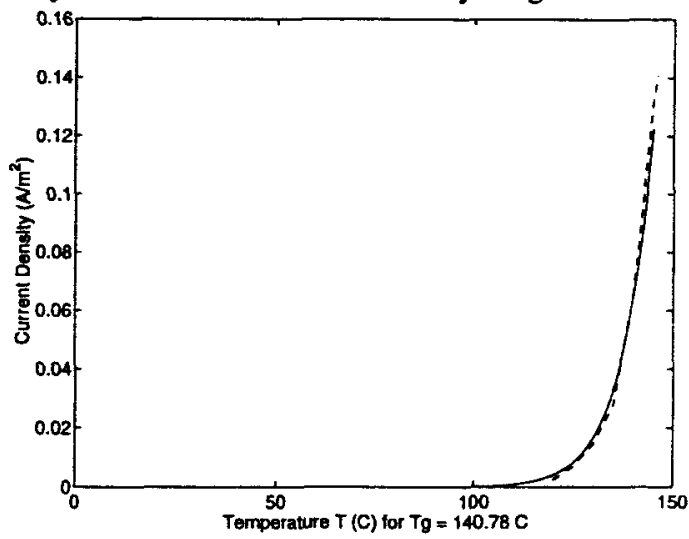

Fig. 7. Model (solid line) vs. Experiment (dashed line) Total Poling Current 
Fig. 7 and 8 shows a comparison between the experimental data and the model results. The experimental results were generated by poling a CLD1/APC core layer with NOA71 upper and lower cladding layers at $500 \mathrm{~V}$ and $145 \mathrm{C}$. The experimental results match the model predictions extremely well during poling as shown in Fig. 7. The small deviations in the curves are due to measurement error. For this poling run, the voltage was turned on after the sample had gone through a 30 minute purge at $120 \mathrm{C}$ to remove trapped oxygen. The voltage was then maintained back down to $30 \mathrm{C}$.

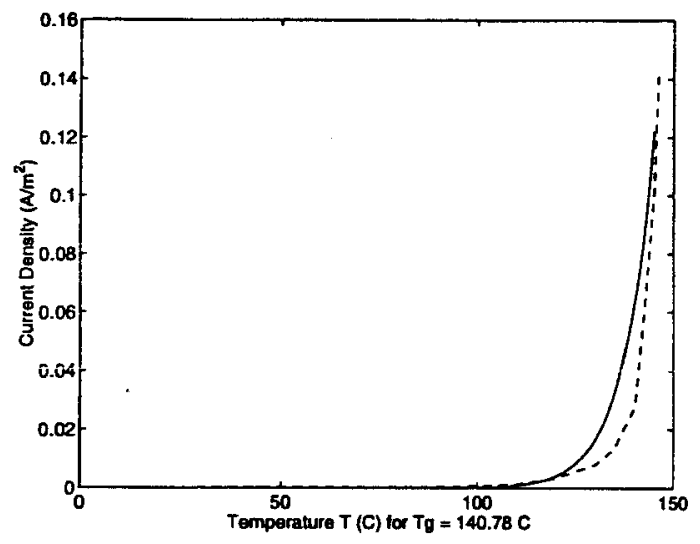

Fig. 8: Boundary Charge Density (solid line) and Polarization Charge Density (dashed line) during dwell at $145 \mathrm{C}$.

During cool down, the experimental data varies more as shown in Fig. 8. This shows the sensitivity to the cool down rate. During the experiment, the cooling rate is initially much slower than $14 \mathrm{C} / \mathrm{min}$ rate used for the model and increases with decreasing temperature till a peak is reached at approximately $120 \mathrm{C}$ which is higher than the average rate used in the model. The faster cool down rate clearly causes a much faster drop in current as compared to the model.

Various factors can be immediately seen from the model. Equations (13) and (14) show that the maximum poling charge density is directly proportional to poling field. Thus, the level of poling achieved is directly related to the magnitude of the poling field. For CLD1 poling temperatures cannot be raised much past $160 \mathrm{C}$ or the chromophore molecules begin to degrade. However, this can be compensated by extending the poling dwell time. By slowing the heating and cooling rates, or dwelling at the maximum poling temperature for longer time periods, much higher poling efficiencies can be obtained as illustrated in Fig. 9.

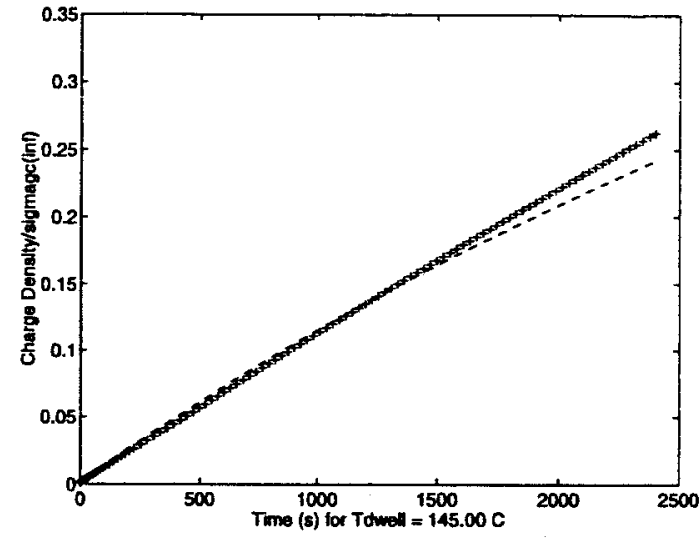

Fig. 9: Model (solid line) vs. Experiment (dashed line) Total Cool Down Current

While the poling temperature is maintained at a constant $145 \mathrm{C}$, the boundary charge and polarization charge density continue to increase. Note that for a 20 minute dwell the charge increases from .011 to .262 of the maximum charge achievabie as defined by (13) and (14). This indicates that the boundary charge has a role in poling mechanisms. The boundary charge role can be considered a part of the dipole orientation directing field. This is easily seen be relating the Onsager Equation [17] to (12) where the directing field and can be taken as:

$$
E_{D}=\left(\frac{V_{P}}{h_{c}}+\frac{\sigma_{c g}(t)}{\varepsilon_{0} \varepsilon_{c}}\right)
$$

Comparing Fig.s 4 and 9 also shows that no significant poling improvements are realized by increasing the poling temperature from $145 \mathrm{C}$ to $160 \mathrm{C}$ (maximum survivable temperature for CLD) relative to increased dwell times.

Another important factor revealed by the model is the large boundary charge density. Electrostriction, while desirable in some materials, constricts the waveguide preventing light propagation. Clearly, boundary charge density can play a large role in electrostriction by creating a permanent electric field which can compress some materials.

\section{SUMMARY}

Based on charge densities and current densities, a model has been developed to describe the poling mechanisms for a 3 layer optical waveguide structure. The model correlates well to experimental results. The model shows that there is a maximum relative efficiency linearly related to the poling field. For a given field a maximum polarization is 
achievable which may be less than the total chromophore available for alignment. The modeil also shows that poling rate and dwell times can greatly increase the poling efficiency. The boundary charge can also have an effect on the formation of electrostriction in some waveguide structures, destroying light propagation.

\section{REFERENCES}

[1] Sessler, G. M., ed., Electrets, $2^{\text {nd }}$ ed., Springer-Verlag, Berlin, 1987.

[2] Prasad, P. N., Williams, D., J., Introduction to Nonlinear Optical Effects in Molecules and Polymers, Wiley, NY, 1991.

[3]Chemla, D. S., Zyss, J., Nonlinear Optical

Properties of Organic Molecules and Crystals, Volume 1, Academic Press, Orlando, 1987, pp. 31-105.

[4] Turnhout, J., Thermally Stimulated Discharge of Polymer Electrets, NY, Elsevier, 1975, pg. 27, 40, 138 - 149.

[5] Sessler, G. M., "Charge Dynamics in Irradiated Polymers",IEEE 9th International Symposium on Electrets (ISE9), 1996, pp. 23 42.

[6] Sessler, G. M., Figueiredo, M., T., Ferreira, G. F. L., "Models of Charge Transport in Electron-Beam Irradiated Insulators", IEEE 11th International Symposium on Electrets (ISE1 1), 2002 , pp. $23-42$.

[7] Bauer-Gogonea, S., Cheng, Z.-Y., Wirges, W., Bauer, S., Gerhard-Multhaupt, R., DasGupta, D. K., "Dielectric Investigation of Thermally-induced Chromophore Degradation in Nonlinear Optical Polymer Electrets", IEEE Trans. Dielec. Elec. Ins., vol. 5, no. 1, Feb 1998, pp. 21 - 25.

[8]Bauer-Gogonea, S., Gerhard-Multhaupt, R., "Nonlinear Optical Polymer Electrets Current Practice", IEEE Trans. Dielec. Elec. Ins., vol. 3, no. 5, Oct 1996, pp. $677-705$.

[9] Bauer, S., Bauer-Gogonea, S., "Current Practice in Space Charge and Polarization Profile Measurements Using Thermal Techniques", IEEE Trans. Dielec. Elec. Ins., vol. 10, no. 5 , Oct 2003, pp. 883-902.

[10] Sessler, G. M., "Charge Distribution and Transport in Polymers", IEEE Trans. Dielec. Elec. Ins., vol. 4, no. 5, Oct 1997, pp. 614-628. [11] Bauer, S., Ren, W., Bauer-Gogonea, S., Gerhard-Multhaupt, R., Liang, J., Zyss, J., Ahlheim, M., Stahelin, M., Zysset, B., "Thermal Stability of the Dipole Orientation in Nonlinear
Optical Guest-Host, Side-Chain and CrossLinked Polymer Electrets", IEEE, 1994, pp. 800 -805 .

[12] Borisova, M. E., Marchenko, M. S., "Influence of Irradiation on the Thermally Stimulated Depolarization Currents of Polyimide Films", $10^{\text {th }}$ International Symposium on Electrets, IEEE, 1999, pp. 781 - 784.

[13] Koykov, S. N., Panteleev, Y. A., "Computer Simulation of TSP and TSD Current in Dielectrics on the Basis of Some Physical Models of Charge Relaxation", $10^{\text {th }}$ International Symposium on Electrets, IEEE, 1999, pp. 423 426.

[14] Grote, J. G., Zetts, J. S., Nelson, R. L., Hopkins, F. K., Dalton, L. R., Zhang, C., Steier, W. H., "Effect of Conductivity and Dielectyric Constant on the Mdoulation Voltage for Optoelectronic devcives based on nonlinear optical polymers", Opt. Eng., No. 40, Vol 11, Nov. 2001, pp. 2464-2473,

[15] Ren, W., Bauer, S., Yilmaz, S., Wirges, W., Gerhard-Multhaupt, R., "Optimized poling of nonlinear optical polymers based on dipoleorientation and dipole-relaxation studies", $J$. Appl. Phys., Vol. 75, No. 11, 1 June 1994, pp. 7211 - 7219 .

[16] Kohler, W., Robello, D. R., Dao, P., T., Willand, C. S., Williams, D. J., "Second harmonic generation and thermally stimulated current measurements: A study of some novel polymers for nonlinear optics", J. Chem. Phys., Vol. 93, No. 12, 15 Dec 1990, pp. 9157 - 9166. [17] Bottcher, C. J. F., Van Belle, O. C., Bordwijk, P., Rip, A., Theory of Electric Polarization: Vol. 1 Dielectrics in Static Fields, NY, Elsevier, 1973, pp 165, 178.

Michael D. Watson was born in Lexington, Kentucky in 1964. He earned a BSEE from the University of Kentucky in 1987 and a MSE in Electrical and Computer Engineering majoring in optics at the University of Alabama in Huntsville in 1996.

He has worked as an engineer and optical physicist for the NASA Marshall Space Flight Center since 1989. Prior to this he served in the United States Air Force in 1988.

1996. 